\title{
Safety of screening with Human papillomavirus testing for cervical cancer at three-year intervals in a high-risk population: experience from the LAMS study*
}

\author{
S F Derchain, LO Sarian, P Naud, C Roteli-Martins, A Longatto-Filho, S Tatti, \\ M Branca, M Eržen, L Serpa-Hammes, J Matos, R C Gontijo, J F Bragança, T P Lima, \\ M Y S Maeda, A Lörincz, G B Dores, S Costa, S Syriänen and K Syriänen
}

J Med Screen 2008;15:97-104

DOI: 10.1258/ims.2008.007061

See end of article for authors' affiliations

....................

Correspondence to: Luís Otávio Sarian, Alexander Fleming, 848, Nova Campinas, Campinas, São Paulo, Brazil;

sarian@terra.com.br

Accepted for publication 17 March 2008

\begin{abstract}
Objectives To assess whether human papillomavirus (HPV) testing is a safe enough approach to warrant extension of the screening intervals of baseline Papanicolaou (Pap)-/HPV - women in low-income settings.

Methods Of the > 1000 women prospectively followed up as part of the Latin American Screening (LAMS) Study in São Paulo, Campinas, Porto Alegre) and Buenos Aires, 470 women with both baseline cytology and Hybrid Capture $2(\mathrm{HC} 2)$ results available were included in this analysis. These baseline Pap-negative and $\mathrm{HC} 2$ - or $\mathrm{HC} 2+$ women were controlled at six-month intervals with colposcopy, HC2 and Pap to assess the cumulative risk of incident Pap smear abnormalities and their predictive factors.

Results Of the 470 women, 324 (68.9\%) were high-risk HPV (hrHPV) positive and 146 (31.1\%) were negative. Having two or more lifetime sex partners lodds ratio [OR] $=2.63 ; 95 \% \mathrm{Cl} 1.70-$ $3.51)$ and women using hormonal contraception $(O R=2.21 ; 95 \% \mathrm{Cl} 1.40-3.51)$ were at increased risk for baseline hrHPV infection. Baseline hrHPV+ women had a significantly increased risk of incident abnormal Pap smears during the follow-up. Survival curves deviate from each other starting at month 24 onwards, when hrHPV + women start rapidly accumulating incident Pap smear abnormalities, including atypical squamous cells (ASC) or worse (log-rank; $P<0.001$ ), lowgrade squamous intraepithelial lesions (LSIL) or worse $(P<0.001)$ and high-grade squamous intraepithelial lesions (HSIL) $(P=0.03)$. Among the baseline hrHPV - women, the acquisition of incident hrHPV during the follow-up period significantly increased the risk of incident cytological abnormalities (hazard ratio $=3.5 ; 95 \% \mathrm{Cl} 1.1-11.7$ ).

Conclusion These data implicate that HPV testing for hrHPV types might be a safe enough approach to warrant extension of the screening interval of hrHPV - /Pap-women even in low-resource settings. Although some women will inevitably contract hrHPV, the process to develop HSIL will be long enough to enable their detection at the next screening round (e.g. after three years).
\end{abstract}

\section{INTRODUCTION}

B ased on firm documentation of efficacy and feasibility, the combined use of cervical cytology and high-risk human papillomavirus (hrHPV) testing has been proposed as an optional approach in screening of cervical cancer in the USA. ${ }^{1-5}$ One of the advantages of this approach includes the possibility of lengthening the screening intervals to three years or more, because women with a negative HPV test and normal cytology are at an extremely low risk of developing cervical cancer in the next three to five years. ${ }^{3}$

A series of large cohort studies to provide evidence on the safety of screening HPV-negative women at three-year intervals are ongoing, using various study designs. ${ }^{6}$ Until to date, the bulk of the current evidence favouring the extended screening intervals is derived from developed countries with effective screening programmes. ${ }^{7}$ In low-resource

* LAMS (Latin American Screening) Study, funded by European Commission, INCO-DEV Contract \# ICA4-CT-2001-10013. settings, the main efforts have been focused on finding the ways how to overcome the difficulties in cervical cytology, by running cross-sectional studies to compare optional screening tools, e.g. visual inspection with acetic acid (VIA)/lugol iodine (VILI) and cervicography. ${ }^{8,9}$ Longitudinal cohort studies capable of confirming the safety of extended screening intervals, based on hrHPV testing or any other screening techniques, are currently not available in the lowresource settings.

In a recent meta-analysis of the prospective studies on HPV tests as a predictor of cervical disease, it has been shown that the predictive value of HPV tests is largely dependent on the disease prevalence in each setting, which precludes extrapolation of the results to populations with different disease burden e.g. in different regions of Latin America. ${ }^{7}$ In Brazil and Argentina (the two largest Latin American countries), screening for cervical cancer is based on cervical cytology but flawed due to several reasons. Most notably, these failures are a consequence of the lack of structured network of public health services, 
the extent of these countries' territories and the geographic dispersion of their populations. ${ }^{10,11}$ For these reasons, Brazil and Argentina are fertile grounds for studies testing optional measures for cervical cancer screening.

This prompted us to design the Latin American Screening Study (LAMS Study), a multicentre collaborative trial evaluating eight different screening techniques for cervical cancer in different regions of Brazil and Argentina. ${ }^{12}$ A cohort of over 12,000 women was enrolled from five different regions, the study design, baseline data and tentative results of hrHPV testing and VIA/VILI being reported. ${ }^{13,14}$

Based on the completion of the prospective follow-up of over 1000 of these women, ${ }^{13}$ the present study reports the acquisition of cytological abnormalities among baseline hrHPV-positive and hrHPV-negative women, derived from this low income, relatively unassisted and previously incompletely studied Latin American population. The main aim was to assess, whether hrHPV testing is a safe enough approach to warrant extension of the screening intervals of those women who test Papanicolaou (Pap)- and hrHPV-negative at baseline. If applicable, such an extension would be of great interest to health authorities planning new measures for cervical cancer prevention in a setting with limited health-care resources.

\section{SUBJECTS AND METHODS}

\section{Study design}

LAMS study is a multicentric study, sponsored by the European Commission through its INCO-DEV programme (ICA4-CT2001-10013). In this study, consecutive women from the cities of Campinas, São Paulo and Porto Alegre (Brazil) as well as Buenos Aires (Argentina) were recruited to undergo gynaecological examination and testing with conventional Pap smear, VIA or VILI, cervicography and screening colposcopy. Women were sampled for hrHPV testing by Hybrid Capture 2 (HC2). All centres performed conventional Pap smear, HC2 and VIA, whereas Porto Alegre performed VILI, Buenos Aires did screening colposcopy and Campinas did cervicography. ${ }^{13,14}$ The study protocol has been approved by the local Ethics Committees of all participating clinics. All enrolled women gave their agreement to participate by signing the informed consent forms, written in their native language.

\section{Study centres and their demographics}

The study design and features of the clinical centres as well as the demographics of their regions were described in detail recently. ${ }^{13,14}$ In brief, Campinas and São Paulo are two large south-western Brazilian cities, located only $100 \mathrm{~km}$ apart, with equivalent standards of living. Cervical cancer is the fourth major cause of cancer death among women, accounting for $3.3 \%$ of all female deaths due to cancer. ${ }^{11}$ The third Brazilian partner is from Porto Alegre, located south of the country. This region offers the best quality of life in Brazil, and cervical cancer is the sixth major cause of cancer death, accounting for $6.1 \%$ of all female deaths due to cancer. ${ }^{11}$ The Argentine partner is from Buenos Aires. The country has an overall cervical cancer mortality rate of $7.6 / 100,000$ women, ${ }^{10}$ but most of the national statistics pertain to Buenos Aires city only.

\section{Enrolment and eligibility of the women}

Slightly different protocols were used to recruit the women in different clinics. In São Paulo, Porto Alegre and Buenos Aires, eligible women were informed of the study protocol by their local health units, inviting them to participate. In Campinas, in addition to this same approach, students and employees of the University Hospital were invited through an open announcement, widely distributed in the university facilities.

Women were considered eligible, if they met all of the following criteria: (1) age between 15 and 60 years; (2) no previous surgical procedure of the cervix or corpus; (3) had no history of abnormal Pap test in the past year; (4) free of known current genital condyloma (external or in the cervix), cervical intraepithelial neoplasia (CIN) or cervical carcinoma; (5) had no sexual intercourse during the three days prior to the examination; (6) did not have any confirmed or clinically suspect immunosuppression (HIV, or other conditions that might compromise the immune system).

\section{Diagnostic setting}

After signing the informed consent, women undertook a questionnaire addressing clinical and epidemiological risk factors of HPV, CIN and cervical cancer. All women underwent thorough pelvic examination in this sequence: (1) collection of the Pap smear, (2) collection of HC2 sample and (3) VIA. In Porto Alegre, most women underwent VILI shortly after VIA. All women, who had at least one of these examinations abnormal, were referred for colposcopic examination. In Buenos Aires and Campinas, women underwent screening colposcopy even when their exams were negative. Abnormal colposcopy prompted punch biopsies of the cervix, and women with high-grade squamous intraepithelial lesions (HSIL) were treated by conization.

Women had their second visit scheduled for one and a half months (average 45 days), to become informed about their exam/biopsy results and to be allotted to either (1) the treatment- or (2) the follow-up group. Treatment was offered to all women who had high-grade lesion (CIN2-3) confirmed in the cervical biopsy. Altogether, 32 cases of invasive cervical cancer were diagnosed during the recruitment phase and all were treated according to each institution's regular protocols.

\section{Follow-up}

A total cohort of 1011 women completed at least one follow-up visit. In the present study, however, we analysed the follow-up data derived from a cohort of 689 women, who attended at least one follow-up visit and who had both baseline cytology and HC2 results available. This group was further reduced to 470 , because 219 women had ASC (atypical squamous cells) or worse in their baseline cytology or CINl or worse in their baseline cervical biopsy. Women with these conditions were treated according to the study protocol as described elsewhere. ${ }^{14}$ Therefore, the study sample in the present study consists of 470 women with negative baseline cytology and normal colposcopy and/or normal biopsies, who attended at least one follow-up visit.

The follow-up workup for women considered 'normal' after baseline assessment was similar in all study centres, with follow-up visits being scheduled at six-month intervals. The study protocol determined that women should be 
re-examined four times (optimal moments should have been 6, 12, 18 ad 24 months; see below). At each follow-up visit, women responded to a brief questionnaire addressing any relevant gynaecological events and epidemiological features changed since the previous control (e.g. sexual partners, smoking). The total follow-up time encompassed in this report approaches 50 months, but the bulk of the data covers approximately 36 months (median follow-up time $=24.4$ months; $90 \%$ central range $=6.8-32.2$ months) (Figure 1).

\section{Difficulties with follow-up}

Longitudinal studies in low-income settings are known to be affected by severe loss-to-follow-up phenomena. The researchers tried to keep consultations as close as possible to these target points, but several factors made it impossible to maintain follow-up on schedule. First and foremost, difficulties to contact women that had missed one of the follow-up consultations led to a delay on several scheduled consultations. Most women in the present study belong to low-income populations, and therefore home addresses and telephone contact numbers fluctuate over time. Table 1 shows the distribution of women at each 'target' follow-up point according to the actual time point at which they attended consultations. The researchers tried to circumvent the patients' difficulties to attend consultations by offering transportation to the clinics and by re-scheduling consultations at the patients' conveniences. Follow-up therefore ranged from 6 to 49 months, although the bulk of data covers 36 months.

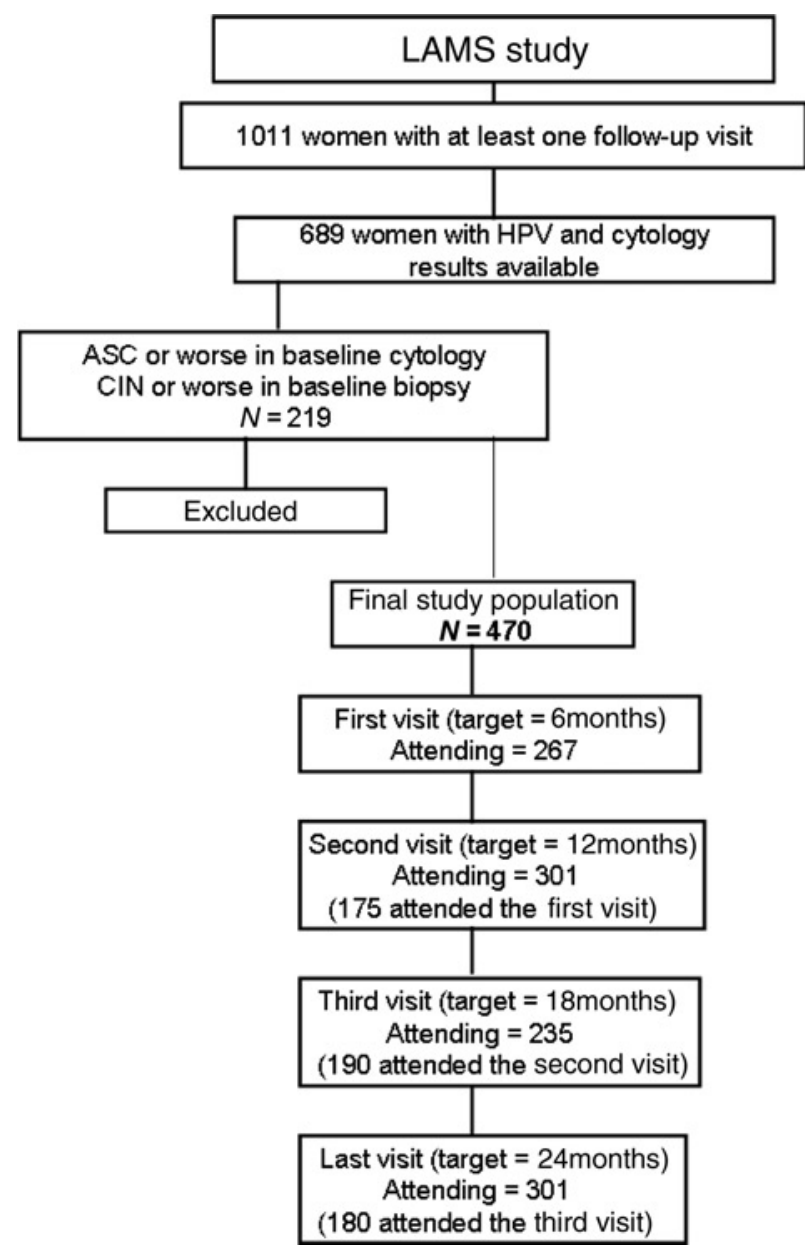

Figure 1 Schematic overview of the study design
Table 1 Time-related distribution of the women that attended follow-up visits

\begin{tabular}{|c|c|c|c|c|c|c|}
\hline & \multicolumn{5}{|c|}{$\begin{array}{l}\text { Follow-up time periods } \\
\text { (in months) }\end{array}$} & \multirow{2}{*}{$\begin{array}{l}\text { Numbe } \\
\text { of case }\end{array}$} \\
\hline & Min & $25 \%$ & $50 \%$ & $75 \%$ & Max & \\
\hline $\begin{array}{l}\text { First visit } \\
\text { Target }=6 \text { months }\end{array}$ & 5.4 & 6.2 & 6.4 & 7.6 & 9.1 & 267 \\
\hline $\begin{array}{l}\text { Second visit } \\
\text { Target }=12 \\
\text { months }\end{array}$ & 11.7 & 12.2 & 13.2 & 15.4 & 17.5 & 301 \\
\hline $\begin{array}{l}\text { Third visit } \\
\text { Target }=18 \\
\text { months }\end{array}$ & 17.8 & 18.2 & 19.0 & 21.3 & 23.4 & 235 \\
\hline $\begin{array}{l}\text { Last visit } \\
\text { Target }=24 \\
\text { months }\end{array}$ & 23.9 & 24.4 & 26.2 & 29.5 & 49.1 & 301 \\
\hline Complete cohort & 5.4 & 9.8 & 17.6 & 24.4 & 49.1 & 470 \\
\hline
\end{tabular}

\section{Cervical cytology (pap smear)}

Conventional Pap smears were taken using the Ayre spatula and endocervical brush, fixed in $95 \%$ ethanol and stained by the modified Pap method. Final cytological diagnoses were issued by using the Bethesda System ${ }^{15}$ and were classified as normal/inflammatory, ASC, atypical glandular cells, LSIL, HSIL or cancer.

\section{Hybrid capture 2}

The specimens for HC2 were tested with probe B for hrHPV types: HPV16, 18, 31, 33, 35, 39, 45, 51, 52, 56, 58, 59 and $68)^{16}$ and the tests were classified positive at the relative light unit/positive control (RLU/CO) ratio of $1 \mathrm{pg} / \mathrm{mL}$ or greater. These RLU/CO ratios provide a semi-quantitative estimate of the amount of HPV DNA in the specimens, i.e. the viral load in the sample. Storage of specimens and reagents, as well as exams processing, were carried out in manufacturer-certified laboratories, under the responsibility of the investigators, following the manufacturer's instructions (Digene Diagnostics Inc., USA). São Paulo and Buenos Aires processed their own HC2 samples in-house, whereas Campinas and Porto Alegre had their HC2 specimens processed at Campinas University hospital laboratory.

\section{Statistical analysis}

All statistical calculations were performed with the R-environment for statistical computing, ${ }^{17}$ and 95\% CI were calculated where appropriate. Intra-class correlation coefficients were calculated to assess the agreement (i.e. re-incidence or repetition of Pap abnormalities) in pairs of cytological assessment rounds. A logistic regression model was used to analyse the power of epidemiological (and clinical) variables as predictors of the baseline hrHPV status, calculating odds ratio (OR) and 95\% CI. Multivariate survival (Cox proportional hazards) analysis was used to calculate hazard ratios (HR) for incident Pap smear abnormalities in three distinct cohorts: (1) the complete sample; (2) women with positive baseline hrHPV; (3) women with negative baseline hrHPV, adjusted for clinical and epidemiological features as well as their hrHPV status at baseline and during follow-up. Univariate survival (Kaplan-Meier) analysis was used to calculate the survival curves (in the whole cohort) for accumulation of incident HSIL during the follow-up, separately for baseline hrHPV-positive 
and -negative women. For cases of ASC or worse and LSIL or worse, the patients' status at their last follow-up visit was considered. This strategy was implemented to avoid censoring cases with transient conditions, such as ASC or LSIL, which would afterwards develop more severe cytological abnormalities or simply subside. The curves were compared using log-rank (Mantel-Cox) statistics.

\section{RESULTS}

Table 2 summarizes the predictors of baseline hrHPV status in multivariate regression analysis. Of the 470 women, 324 $(68.9 \%)$ were hrHPV positive and $146(31.1 \%)$ were negative. Having two or more lifetime sex partners $(\mathrm{OR}=2.63$; 95\% CI 1.70-3.51) and women using hormonal contraception $(\mathrm{OR}=2.21 ; 95 \% \mathrm{CI} 1.40-3.51)$ were at increased risk for baseline hrHPV infection. Other implicated risk factors of hrHPV, e.g. age at first intercourse and smoking habits, were not significant predictors of baseline hrHPV status in this cohort.

The vast majority of incident cytological abnormalities diagnosed during the follow-up were found among women who tested hrHPV positive at baseline. However, the prevalence of incident ASC, LSIL and HSIL at three follow-up time points (6-, 12-, 24-months) did not differ among hrHPV + and hrHPV - women. At six months, 41 women had newly diagnosed abnormal Pap tests, only one of those being hrHPV - at baseline. However, most of the women $(191 / 224)$ who attended the six-month follow-up visit had a positive baseline hrHPV test. At 12- and 24-month visits, women with positive baseline hrHPV still represented the majority among those with incident Pap smear abnormalities (data not shown).

The actual time points at which women attended the follow-up consultations are depicted, in quartiles, in Table 1 . The number of cases at each visit ranged from a minimum of 235 (third visit, planned to target women 18 months after baseline assessment) to a maximum of 301 (reached at the second [12 months] and last [24 months] visits). Although 470 women attended at least one follow-up visit, attendance varied as follows: 174 attended one

Table 2 Predictors of the baseline hrHPV status in multivariate analysis

\begin{tabular}{|c|c|c|c|c|}
\hline \multirow[b]{2}{*}{ Characteristic } & \multicolumn{4}{|c|}{ Baseline hrHPV } \\
\hline & $\begin{array}{l}\text { Positive } \\
(n=324)\end{array}$ & $\begin{array}{l}\text { Negative } \\
(n=146)\end{array}$ & $\mathrm{OR}^{*}$ & $95 \% \mathrm{Cl}^{*}$ \\
\hline \multirow{3}{*}{$\begin{array}{l}<30 \\
\geq 30 \\
\text { First intercourse } \\
\quad<18 \text { years } \\
\quad \geq 18 \text { years } \\
\text { Life time partners } \\
\quad \geq 2 \\
\quad 1\end{array}$} & $\begin{array}{l}141(43.5) \\
183(56.5)\end{array}$ & $\begin{array}{l}57(39.6) \\
87(60.4)\end{array}$ & 0.80 & $\begin{array}{l}\text { (0.23-1.28) } \\
\text { Ref. }\end{array}$ \\
\hline & $\begin{array}{l}181(55.8) \\
143(44.2)\end{array}$ & $\begin{array}{l}66(45.2) \\
80(54.8)\end{array}$ & 1.37 & $\begin{array}{l}\text { (0.88-2.13) } \\
\text { Ref. }\end{array}$ \\
\hline & $\begin{array}{r}234(72.2) \\
90(37.8)\end{array}$ & $\begin{array}{l}74(50.7) \\
72(49.3)\end{array}$ & 2.63 & $\begin{array}{l}\text { (1.70-3.51) } \\
\text { Ref. }\end{array}$ \\
\hline \multicolumn{5}{|c|}{ Contraceptive methods } \\
\hline $\begin{array}{l}\text { Hormonal } \\
\text { Without } \\
\text { Non-hormonal }\end{array}$ & $\begin{array}{r}144(44.6) \\
42(13.0) \\
137(42.4)\end{array}$ & $\begin{array}{l}42(28.8) \\
17(11.6) \\
87(59.6)\end{array}$ & $\begin{array}{l}2.21 \\
1.88\end{array}$ & $\begin{array}{l}(1.40-3.51) \\
(0.97-3.65) \\
\text { Ref. }\end{array}$ \\
\hline $\begin{array}{c}\text { Smoking } \\
\text { Yes } \\
\text { Past } \\
\text { Never }\end{array}$ & $\begin{array}{r}76(23.4) \\
49(15.2) \\
199(61.4)\end{array}$ & $\begin{array}{l}37(25.3) \\
22(15.1) \\
87(59.6)\end{array}$ & $\begin{array}{l}0.69 \\
0.90\end{array}$ & $\begin{array}{l}(0.42-1.16) \\
(0.49-1.64) \\
\text { Ref. }\end{array}$ \\
\hline
\end{tabular}

$\mathrm{OR}$, odds ratio; HrHPV, high-risk HPV

*Adjusted with logistic regression follow-up visit, 84 attended two, 82 attended three visits and 130 completed the follow-up programme (data not shown).

Table 3 depicts the incidence of cytological abnormalities at each follow-up visit as a function of the Pap results in previous exams. HSIL was detected in exams collected at the first, third and last follow-up visits. Women that were found to have HSIL at one given visit are reported to be 'missing' in the next ones; however, they were excluded from the normal follow-up schedule, treated, and allotted to another follow-up series, not under the scope of the present report. The intra-class correlation coefficient (ICCs) calculated for each pair of follow-up consultations varied between 0.135 (second versus third visits) and 0.279 (second versus last), revealing that cytological results agreed (i.e. repetition of cytological abnormalities) the best considering the 12 months and the final assessment of the women. However, the clinical relevance of these figures is questionable: it must be noted that the 12 and 24-month visits were the most attended (301 women at each) and the higher ICC for the comparison of these two rounds is much likely attributable to the fact that more women attended both these consultations as compared with any of the other 'visit X visit' tabulations.

Figure 2 summarizes the cumulative incidence of new abnormal Pap smears among baseline hrHPV+ and hrHPV - women in univariate (Kaplan-Meier) analysis. Clearly, baseline hrHPV+ women had a significantly increased risk of incident abnormal Pap smears during the follow-up. Survival curves deviate from each other starting at month 24 onwards, when hrHPV + women start rapidly accumulating incident Pap smear abnormalities, including ASC or worse (log-rank; $P<0.001$ ), LSIL or worse $(P<0.001)$ and HSIL $(P=0.03)$.

The risk estimates for incident Pap smear abnormalities during a 24-month follow-up period in Cox analysis are depicted in Table 4. Positive baseline hrHPV status $(\mathrm{HR}=3.4 ; 95 \% \mathrm{CI} 1.8-6.4)$ and age at first intercourse below 18 years $(\mathrm{HR}=1.9 ; 95 \% \mathrm{CI} 1.2-3.0)$ were the two significant predictors of incident cytological abnormalities in the present (whole) cohort. Among the sub-cohort of baseline hrHPV + women, none of the recorded epidemiological variables were shown to be significant predictors. Among the baseline hrHPV - women, the acquisition of incident hrHPV during the follow-up period proved to increase the risk of incident cytological abnormalities $(\mathrm{HR}=3.5 ; 95 \%$ CI $1.1-11.7)$.

\section{Discussion}

During the past few years, HPV detection technology has become standardized and cost-effective, and HPV tests have recently gained increasing importance as potential tools in cervical cancer screening. ${ }^{3,6}$ HPV testing has an almost 100\% negative-predictive value for the absence of significant cervical lesions when the test is negative. This has prompted several professional societies, e.g. the American Cancer Society (ACS) and the American College of Obstetrics and Gynecology (ACOG) to issue guidelines recommending a combined use of HPV tests with cytology. Useful as these guidelines might be in high-resource settings, it is imperative to realize that these are based on the epidemiological profile of the populations where HPV testing has been shown to predict future cervical abnormalities, which is not necessarily the case in many low-resource settings. ${ }^{18,19}$ 
Table 3 Cytological abnormalities at each follow-up visit according to the results of a previous follow-up cytology

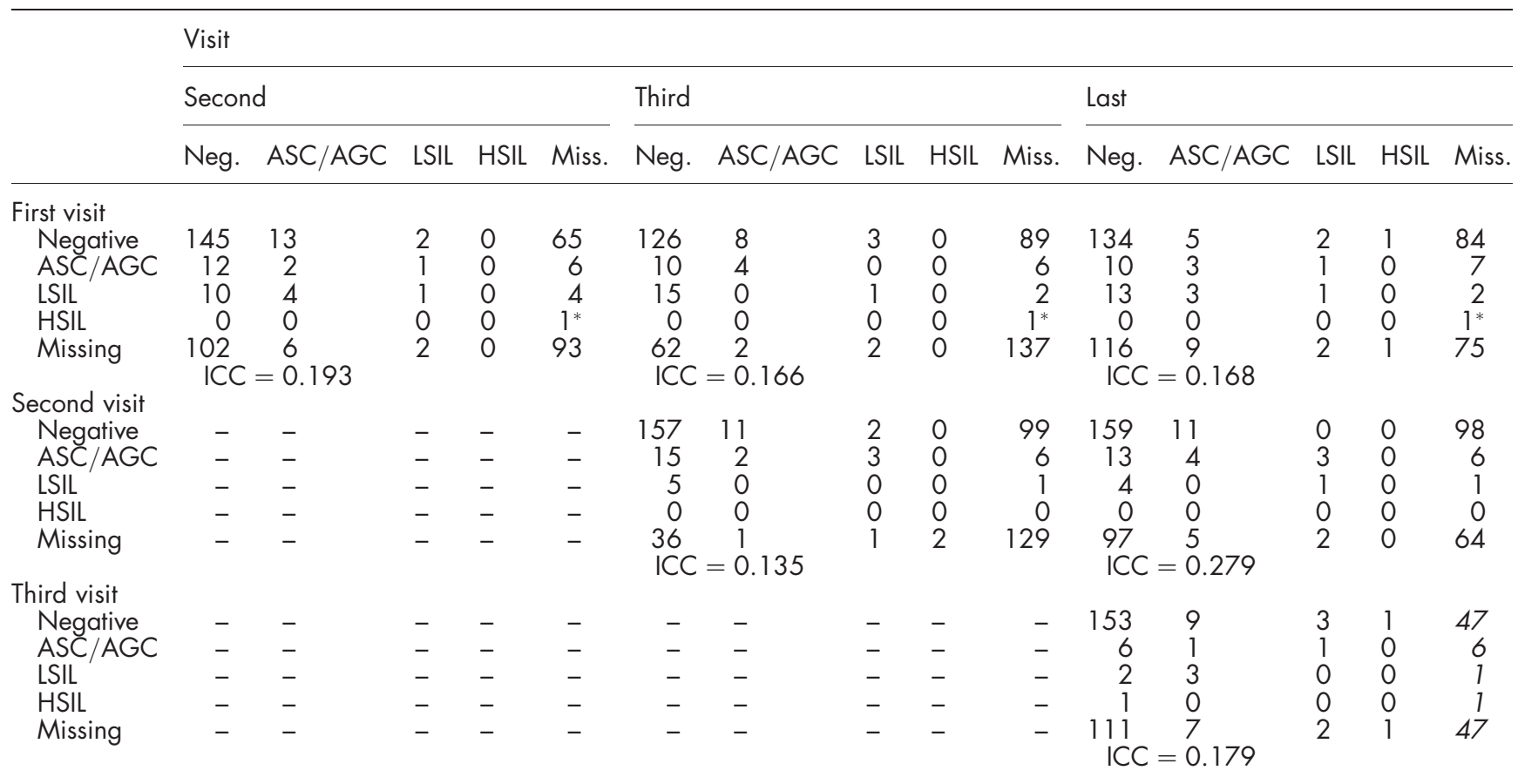

Neg., negative (normal); Miss., missing (not attending) that specific follow-up visit; ICC, intra-class correlation coefficient (agreement); ASC, atypical squamous cells; LSSL, low-grade squamous intraepithelial lesions; HSIL, high-grade squamous intraepithelial lesions

*One patient treated with hysterectomy and excluded from follow-up

While testing optional screening tools in low-resource settings, our LAMS study provides data that enables tackling the validity of these ACS and ACOGs guidelines in another type of environment, where the prevalence of HPV infection and cervical cancer are dramatically different. Importantly, several co-factors are needed to make hrHPV infections capable of producing clinically significant cervical disease (CIN2-3), and these potential co-factors (e.g. reproductive factors, sexual behaviour, smoking, nutrition, concomitant gynaecological infections) are known to differ substantially among women living in high-and low-resource settings. ${ }^{20,21}$ Thus, if HPV testing is to be proposed as a suitable screening tool for these unprivileged conditions, the performance of this technology needs to be established in prospective cohort studies conducted under field conditions in these particular. ${ }^{6,7}$ This is exactly what the LAMS study has done, and the data provided in the present study should constitute an important tool for health policy-makers, while confirming the significant predictive value of hrHPV test as determinant of incident Pap smear abnormalities also among these lowincome women (Table 1; Figure 1).

The sample of the present study $(n=470)$ represents a selected sub-cohort derived from over 12,000 women examined at baseline in the LAMS study, of whom over 1000 completed at least one follow-up visit. ${ }^{13}$ Despite this selection, this series of patients is consistent with the large crosssectional studies, as to the epidemiological risk factors of cervical disease. ${ }^{6,7}$ In this sub-cohort, the number of lifetime partners and hormonal contraception were closely related to HPV infection, whereas age, age at first intercourse, and smoking were not (Table 2). This is not unexpected, given the fact that this subset of the LAMS cohort analysed in this study was not large enough to give the study a high statistical power to detect minor true differences in these
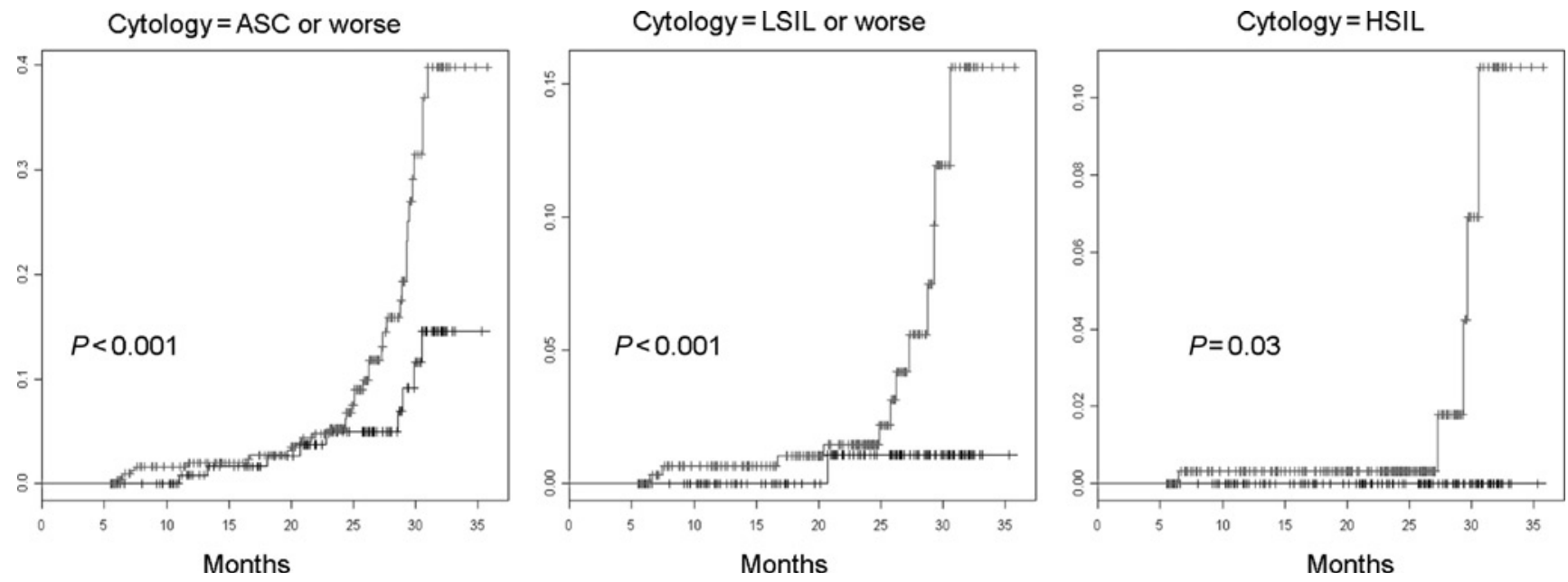

Figure 2 Cumulative incidence of cytological abnormalities during 36 months of follow-up in Kaplan-Meier and log-rank analysis. Baseline hrHPV-positive women are depicted with a dotted line. Note: different scales for the Y (probability) axis were used in each plot 
Table 4 Risk estimates for incident cytological abnormalities (atypical squamous cells, low-grade squamous intraepithelial lesions and high-grade squamous intraepithelial lesions) during follow-up

\begin{tabular}{|c|c|c|}
\hline Characteristic & Whole cohort $n=470$ & $\begin{array}{l}\text { Women with positive baseline } \\
\text { hrHPV } n=324\end{array}$ \\
\hline $\begin{array}{l}\text { Positive baseline } \\
\text { hrHPV }\end{array}$ & $3.4(1.8-6.4) n=324(68.9 \%)$ & - \\
\hline Age $\leq 30$ years & $0.8(0.5-1.3) n=198(42.1 \%)$ & $0.8(0.5-1.3) n=141(43.5 \%)$ \\
\hline $\begin{array}{l}\text { Age at first sexual } \\
\text { intercourse }<18 \\
\text { years }\end{array}$ & $1.9(1.2-3.0) n=247(52.5 \%)$ & $1.6(1.0-2.6) n=181(55.8 \%)$ \\
\hline Life time partners $\geq 2$ & $0.8(0.5-1.3) n=308(65.5 \%)$ & $0.7(0.5-1.3) n=234(72.2 \%)$ \\
\hline $\begin{array}{l}\text { Oral hormonal } \\
\text { contraception* }\end{array}$ & $1.3(0.8-2.1) n=186(39.6 \%)$ & $1.3(0.4-1.3) n=144(44.6 \%)$ \\
\hline Smoking ${ }^{\dagger}$ & $0.9(0.5-1.5) n=113(24.0 \%)$ & $0.7(0.4-1.4) n=76(23.4)$ \\
\hline $\begin{array}{l}\text { Persistent HPV during } \\
\text { follow-up }\end{array}$ & - & $1.5(0.9-2.5) n=103(31.7 \%)$ \\
\hline $\begin{array}{l}\text { Incident HPV during } \\
\text { follow-up } \neq\end{array}$ & - & - \\
\hline
\end{tabular}

epidemiological factors linked to HPV infection. This was not the main focus of this study, however, but instead we wanted to assess the accumulation of cytological abnormalities during the follow-up of baseline hrHPV + and hrHPVwomen. As shown by the significant results, the sample size was clearly large enough for this specific purpose.

Indeed, testing hrHPV positive at baseline significantly increased the probability of contracting incident Pap smear abnormality during the 50 months of prospective follow-up (Table 1). This was true for all cytological cut-offs used, i.e. ASC-US, LSIL and HSIL, all of which were significantly more frequent among baseline hrHPV+ women (Figure 1). As shown by the survival curves in Figure 1, all degrees of cytological abnormalities start accumulating after the first 24 months of prospective follow-up and continue to do so until the end of the 50-month observation period. A similar trend has been demonstrated in several ongoing or concluded cohort studies in Europe as well. ${ }^{2-24}$ In the HART (HPV in Addition to Routine Testing) study, the proposed strategy of reserving cytology exclusively to triage women with positive HPV tests has been severely challenged by the high frequency of low-grade smears among HPV + women. ${ }^{22}$ Interestingly, this happened even though they included only women aged 30-60 years, attempting to exclude most of the low-grade abnormalities due to transient hrHPV - and harmless low-risk HPV infections. $^{22}$ It should be noted that the cytological terminology used in the HART study was not compliant with the Bethesda system. ${ }^{22}$ Another major difference between the HART and LAMS studies is in the living standards of the eligible women, the former also having a significantly better access to organized screening programmes. The same is true also for the 7932 women included in the French cohort. $^{23}$

It should be also emphasized that cervical cancer, although an important public health concern in many European countries, is only the 10th most common cause of cancer deaths among women in that continent. ${ }^{18}$ This is a major deviation from the epidemiological characteristics of the population from which the LAMS study cohort was enrolled. ${ }^{10,11}$ These major differences preclude the generalization of the results obtained from the European trials on HPV testing as a screening tool, for three important reasons: (1) prevalence of specific HPV types may differ; (2) behavioural and epidemiological patterns (including different exposure to HPV, viral loads at sexual intercourse, concurrent gynaecological infections) may be more risky among women living in low socioeconomic conditions. These in turn could facilitate the acquisition and persistence of hrHPV infections and therefore shortening the protected period' between two rounds of HPV screening; (3) most European trials, with randomized assignment of women to different screening strategies, are currently underway, leaving several questions still open. ${ }^{6}$ All these inherently divergent geographic and other unsolved issues emphasize the necessity of a large population-based study in Latin American continent, capable of evaluating the feasibility of HPV-based screening in an appropriate setting.

The present data implicate that in addition to the baseline hrHPV + women, also women who acquired hrHPV infection during the follow-up were at significantly increased risk $(\mathrm{HR}=3.5,95 \% \mathrm{CI} 1.1-11.7)$ of contracting incident Pap smear abnormalities. Similarly, women who tested hrHPV + at baseline but who cleared their infection, still held their elevated risk of incident Pap smear abnormalities. Such patients were quite few, however, compromising the power of the analysis. On the other hand, most of the Pap abnormalities found in women with incident hrHPV infections were low-grade (data not shown in Tables). This is consistent with the known dynamics of HPV infections and their related lesions, where HPV infection always precedes the development of abnormal Pap by several months. ${ }^{25}$ Because of these temporal relationships, Pap smear abnormalities associated with these newly developed hrHPV infections are of lower grade as compared with those who had hrHPV at baseline. However, similar type of results have been reported in those cohort studies conducted in countries with high standards of living and high-resource primary health care. ${ }^{22-24,26-29}$ This is another argument in favour of the concept that extending the screening interval of HPV - /Pap - women is safe; even if they contract an incident hrHPV, the Pap smear abnormality to be detected in the next screening round (e.g. after three years) is likely to be of low-grade only.

Another debated issue in the literature is the type of HPV testing that should be optimal for screening, the response 
still pending. ${ }^{30,31}$ This is due to multiple reasons, not the least due to the fact that HPV testing technology is still evolving. HC2 (Digene Corp, Gaithersburg, MD, USA) was the option found at the time of study design to be the most suitable for the detection of hrHPV infections in this series. Being cost-effective, easy to collect and fairly reproducible, the technique had the approval for clinical use by the US Food and Drug Administration and its Brazilian counterpart (Agência Nacional de Vigilância Sanitária; ANVISA). The major limitation of this technique is the failure to identify the specific HPV types, which would be highly advantageous in this type of study. Because one of the main aims of this study was to compare the validity of HPV testing in Brazilian and Argentinian women, likely to show different patterns of HPV exposure as compared with the European and North-American women studied in the published reports, ${ }^{9,12,13,21}$ the distribution of HPV genotypes in the infected women would be useful.

Prophylactic HPV vaccines are emerging as an appealing strategy for the primary prevention of cervical cancer. Two vaccines are already available; both having as their primary target the hrHPV types 16 and $18 .^{32}$ These two HPV types were responsible for $50 \%$ and $20 \%$ of cervical cancer cases, respectively, in large population-based surveys. ${ }^{33}$ In the HPV vaccine era, it is likely that further attention will be focused on HPV testing, because efficacy of the vaccines seems to be conditioned by the HPV status of the women. ${ }^{34}$

Taken together, the present study provides firm evidence first time also in low-resource settings, suggesting that hrHPV infection (both baseline and incident) is a significant risk factor for incident Pap smear abnormalities within a relatively short follow-up time. Until now, such data were available only in cohort studies performed in developed countries with completely different disease burden and resources for organized cervical cancer screening. This implicates that HPV testing for hrHPV types might be a safe enough approach to be linked with extension of the screening interval of hrHPV - /Pap - women. Even if some of them will inevitably contract an incident hrHPV, the process to develop high-grade cytological abnormality will be long enough to enable detection of this incident abnormal Pap at the next screening round (e.g. after three years), most likely still at the stage of low-grade. This information should have important implications in planning alternative screening strategies particularly in the developing countries, where all resources for public health care are strictly limited.

P Naud, Associate Professor of Gynaecology, Hospital de Clínicas de Porto Alegre, Brazil

C Roteli-Martins, Assistant Gynaecologist, Hospital Leonor M de Barros, São Paulo, Brazil

A Longatto-Filho, Scientific Researcher, Instituto Adolfo Lutz, São Paulo, Brazil, and Life and Health Sciences Research Institute School of Health Sciences, University of Minho, Braga, Portugal

S Tatti, Professor of Gynaecology and Chief of the Colposcopic Clinic, First Chair Gynecology Hospital de Clínicas, Buenos Aires, Argentina

M Branca, Pathologist, Unit of Cytopathology, National Centre of Epidemiology, Surveillance and Promotion of Health, National Institute of Health (ISS), Rome, Italy

M Eržen, Associate Professor of Pathology, SIZE Diagnostic Center, Ljubljana, Slovenia

L Serpa-Hammes, Gynaecologist, Hospital de Clinicas de Porto Alegre, Brazil
}

J Matos, Gynaecologist, Hospital de Clinicas de Porto Alegre, Brazil R C Gontijo, Gynaecologist, Universidade Estadual de Campinas, Campinas, Brazil

J F Bragança, Gynaecologist, Universidade Estadual de Campinas, Campinas, Brazil

T P Lima, Gynaecologist, Hospital Leonor M de Barros, São Paulo, Brazill

M Y S Maeda, Scientific Researcher, Instituto Adolfo Lutz, São Paulo, Brazil

A Lörincz, Scientific Researcher, Digene Corp., Maryland, USA

G B Dores, Scientific Researcher, Digene Brazil, São Paulo, Brazil

S Costa, Associate Professor, Department of Obstetrics and Gynecology, S. Orsola-Malpighi Hospital, Bologna, Italy

S Syriänen, Professor of Oral Pathology, Department of Oral Pathology, Institute of Dentistry, University of Turku, Finland

K Syriänen, Professor, Department of Oncology \& Radiotherapy, Turku University Hospital, Turku, Finland

\section{ACKNOWLEDGEMENTS}

This study has been supported by the European Commission, INCO-DEV Programme (Contract \# ICA4-CT-2001-10013). The generous contribution from DIGENE Inc. (USA) donating the HC 2 tests is gratefully acknowledged.

\section{REFERENCES}

1 Saslow D, Runowiccz CD, Solomon D, et al. American Cancer Society guideline for the early detection of cervical neoplasia and cancer. $C A$ Cancer J Clin 2002:52:342-62

2 ACOG Practice Bulletin. Cervical Cytology Screening. Washington, DC: American College of Obstetricians and Gynecologists, 2003

3 Wright TC, Schiffman M, Solomon D, et al. Interim guidance on the use of HPV DNA testing as an adjunct to cervical cytology. Obstet Gynecol 2004: 103:304-9

4 ACOG Practice Bulletin. Human Papillomavirus. vol 61. April 2005

5 ACOG Practice Bulletin. Management of Abnormal Cervical Cytology and Histology. Clinical Management Guidelines for the Obstetrician and Gynecologist, vol 66, 2005

6 Davies P, Arbyn M, Dillner J, et al. A report on the current status of European research on the use of human papillomavirus testing for primary cervical cancer screening. Int J Cancer 2006;1 18:791-6

7 Koliopoulos G, Arbyn M, Martin-Hirsh P, et al. Diagnostic accuracy of human papillomavirus testing in primary cervical screening: A systematic review and meta-analysis of non-randomized studies. Gynecol Oncol 2007; 104:232-46

8 Cronje HS, Parham GP, Cooreman BF, et al. A comparison of four screening methods for cervical neoplasia in a developing country. Am J Obstet Gynecol 2003; 188:395-400

9 Sodhani P, Gupta S, Sharma JK, et al. Test characteristics of various screening modalities for cervical cancer: a feasibility study to develop an alternative strategy for resource limited settings. Cytopatho 2006; 17:348-52

10 Argentina, Ministry of Health Estadísticas vitales. Información básica año 2005. Buenos Aires: Dirección de Estadísticas e formación de Salud, 2006

11 Ministério da Saúde - Instituto Nacional do Câncer - Atlas de Mortalidade por Câncer no Brasil 1979-1999. INCA, 2002. Available online (only Portuguese) at See [http://www.inca.gov.br/atlas/docs/ Atlas_completo.pdf]

12 Syriänen K, Naud P, Derchain S, et al. Comparing PAP smear cytology, aided visual inspection, screening colposcopy, cervicography and HPV testing as optional screening tools in Latin America. Study design and baseline data of the LAMS study. Anticancer Res 2005;25:3469-80

13 Longatto-Filho A, Erzen M, Branca M, Roteli-Martins C, et al. Human papillomavirus testing as an optional screening tool in low-resource settings of Latin America: experience from the Latin American Screening study. Int J Gynecol Cancer 2006; 16:955-62

14 Sarian LO, Derchain SF, Naud P, et al. Evaluation of visual inspection with acetic acid (VIA), Lugóls lodine (VILI), cervical cytology and HPV testing as cervical screening tools in Latin America. This report refers to partial results from the LAMS study. J Med Screen 2005; 1 2: 142-9

15 Solomon D, Davey D, Kurman R, et al. Forum Group Members; Bethesda 2001 Workshop. The 2001 Bethesda System: terminology for reporting results of cervical cytology. JAMA 2002;287:2114-9

16 Lörincz AT, Castle PE, Sherman ME, et al. Viral load of human papillomavirus and risk of CIN 3 or cervical cancer. Lancet 2002;360:288-9

17 R Development Core Team (2007). R: A language and environment for statistical computing. R Foundation for Statistical Computing, 
Vienna, Austria. ISBN 3-000051-07-0, URL See [http://www.R-project. org]

18 Ferlay J, Bray F, Pisani P, et al. GLOBOCAN: Cancer Incidence, Mortality and PrevalenceWorldwide. Version 1.0. IARC Cancer Base no 5, IARC Press, Lyon, France, 2001

19 Kamangar F, Dores GM, Anderson WF. Patterns of cancer incidence, mortality, and prevalence across five continents: defining priorities to reduce cancer disparities in different geographic regions of the world. J Clin Oncol 2006;24:2137-50

20 Sankaranarayanan R. Overview of cervical cancer in the developing world. FIGO 6th Annual Report on the Results of Treatment in

Gynecological Cancer. Int J Gynaecol Obstet 2006;95:S205-10

21 Wiley D, Masongsong E. Human papillomavirus: the burden of infection. Obstet Gynecol Surv 2006;61:S3 - 14

22 Cuzick J, Szarewski A, Cubie H, et al. Management of women who test positive for high-risk types of human papillomavirus: the HART study. Lancet 2003;362: 1871-6

23 Clavel C, Masure M, Bory JP, et al. Human papillomavirus testing in primary screening for the detection of high-grade cervical lesions: a study of 7932 women. Br J Cancer 2001:84:1616-23

24 Clavel C, Cucherousset J, Lorenzato $M$, et al. Negative human papillomavirus testing in normal smears selects a population at low risk for developing high-grade cervical lesions. Br J Cancer 2004;90:1803-8

25 Syriänen S, Shabalova IP, Petrovichev N, et al. Acquisition of Incident High-Risk Human Papillomavirus (HPV) Infections and PAP Smear Abnormalities in a Cohort of Women Subjected to HPV Screening in the New Independent States (NIS) of the Former Soviet Union. J Clin Microbiol 2004:42:505- 11
26 Clavel C, Masure M, Bory JP, et al. Hybrid Capture II-based human papillomavirus detection, a sensitive test to detect in routine high-grade cervical lesions: a preliminary study on 1518 women. Br J Cancer 1999;80:1306-11

27 Sherman ME, Lorincz AT, Scott DR, et al. Baseline cytology, human papillomavirus testing, and risk for cervical neoplasia: a 10-year cohort analysis. J Natl Cancer Inst 2003;95:46-52

28 Schlecht NF, Kulaga S, Robitaille J, et al. Persistent human papillomavirus infection as a predictor of cervical intraepithelial neoplasia. JAMA 2001;286:3106-14

29 Petry KU, Menton S, Menton M, et al. Inclusion of HPV testing in routine cervical cancer screening for women above 29 years of age in Germany: results for 8466 patients. $\mathrm{Br} J$ Cancer 2003;88: 1570-77

30 Wright TC Jr. Cervical cancer screening in the 21 st century: is it time to retire the PAP smear? Clin Obstet Gynecol 2007;50:313-23

31 Wheeler CM. Advances in primary and secondary interventions for cervical cancer: human papillomavirus prophylactic vaccines and testing Nat Clin Pract Oncol 2007;4:224-35

32 Munoz N, Bosch Lowy DR, Schiller TH. Prophylactic human Papillomavirus vaccines. J Clin Invest 2006; 1 16:1 167-72

33 Bosch FX, Castellsague X, Diaz M, et al. Against which human papillomavirus types shall we vaccinate and screen? The international perspective. Int J Cancer 2004;1 1 1:278-85

34 Schiffman $M$. Integration of human papillomavirus vaccination, cytology, and human papillomavirus testing. Cancer Cytopathol $2007 \cdot 111 \cdot 145-53$ 